\title{
Comportamiento creativo: efectos de la variabilidad y secuencia del entrenamiento
}

\author{
Creative Behavior: Effects of Uariability and Training \\ Comportamento criativo: efeitos da variabilidade e sequência do treinamento
}

\author{
Claudio Carpio, Virginia Pacheco, Germán Morales, \\ Rosalinda Arroyo y Linda Pacheco-Lechón* \\ Universidad Nacional Autónoma de México
}

Doi: dx.doi.org/10.12804/apl33.01.2015.07

\section{Resumen}

Diversos estudios han reportado que, bajo condiciones variables de entrenamiento, el éxito en la transferencia de lo aprendido es mayor que bajo condiciones de entrenamiento constantes. Es viable suponer que, al exponer a los participantes a ambos tipos de entrenamiento y en diferente secuencia, se observarán efectos diferentes a los reportados hasta ahora. En el presente estudio participaron diez estudiantes distribuidos en dos grupos experimentales: Grupo constante-variable (CC-VV) y Grupo variable-constante (VV-CC). Los resultados muestran que, durante el entrenamiento, al margen de la secuencia, los porcentajes de respuestas correctas más elevados se presentan bajo condiciones constantes. En pruebas de transferencia se observa que la transferencia de lo aprendido durante el entrenamiento es baja cuando $\mathrm{CC}$ es el primer entrenamiento recibido. Los resultados en pruebas de comportamiento creativo no arrojaron diferencias significativas entre los grupos, aunque se observa mejor ejecución en el Grupo VV-CC. Los hallazgos amplían la información sobre la contribución de la variabilidad del entrenamiento en la emergencia de comportamiento creativo y adicionalmente se demuestra existencia de reversibilidad y efectos de secuenciación. Palabras clave: Variabilidad en el entrenamiento, secuencia, transferencia, comportamiento creativo

* Claudio Carpio, División de Investigación y Posgrado, Universidad Nacional Autónoma de México, Facultad de Estudios Superiores Iztacala; Virginia Pacheco, División de Investigación y Posgrado, Universidad Nacional Autónoma de México, Facultad de Estudios Superiores Iztacala; Germán Morales, División de Investigación y Posgrado, Universidad Nacional Autónoma de México, Facultad de Estudios Superiores Iztacala; Rosalinda Arroyo, División de Investigación y Posgrado, Universidad Nacional Autónoma de México, Facultad de Estudios Superiores Iztacala; Linda Pacheco-Lechón, División de Investigación y Posgrado, Universidad Nacional Autónoma de México, Facultad de Estudios Superiores Iztacala.

Esta investigación fue posible gracias al apoyo de los programas PAPCA-FESI No. 36, PAPIME PE301512 y PAPIIT RN307013 de la UNAM.

La correspondencia relacionada con este artículo debe ser dirigida a Claudio Carpio, División de Investigación y Posgrado, Av. de los Barrios 1, Col. Los Reyes Iztacala, C.P. 54090, Tlalnepantla de Baz, Estado de México, México. Correo electrónico: carpio@ unam.mx

Para citar este artículo: Carpio, C., Pacheco, V., Morales, G., Arroyo, R., \& Pacheco-Lechón, L. (2015). Comportamiento creativo: efectos de la variabilidad y secuencia del entrenamiento. Avances en Psicología Latinoamericana, 32(3), 91-104. doi: dx.doi.org/10.12804/apl33.01.2015.07 


\section{fibstract}

Several studies have reported that under varying conditions of training, success in the transfer of learning is higher than the transfer observed under constant training conditions. It is viable to assume that the exposition to two different types of training and two different sequences will produce very different effects compared with those found until now. The present study involved two experimental groups of university students: a constant-variable group (CC-VV) and a variable-constant group (VV-CC). Results show that during the training, regardless of the sequence, higher percentages of correct responses are observed under constant conditions. In transfer tests results show that the transfer of learning is lower when constant condition was the first training received. Finally, in creative behavior tests, no significant differences between groups were found, although a better performance was observed in participants from the VV-CC Group. These results contribute to the findings previously found about the effects of the training variability on the emergence of creative behavior and the reversibility of its effects. It was also demonstrated that there is an effect of different presentation of training (sequence).

Keywords: Training variability, sequence, transfer, creative behavior

\section{Resumo}

Diversos estudos têm reportado que sob condições variáveis do treinamento, o sucesso na transferência do aprendido é maior que sob constantes. É viável supor que, ao expor aos participantes a ambos os tipos de treinamento e em diferente sequência, se observarão efeitos diferentes aos reportados até agora. No presente estudo participaram 10 estudantes distribuídos em dois grupos experimentais: Grupo constante-variável (CCVV) e Grupo variável-constante (VV-CC). Os resultados mostram que durante o treinamento, à margem da sequência, as porcentagens de resposta corretas mais elevadas se apresentam sob condições constantes. Em provas de transferência observa-se que a transferência do aprendido durante o treinamento é baixa quando CC é o primeiro treinamento recebido. Os resultados em provas de comportamento criativo não revelaram diferenças significativas entre os grupos, ainda que observa-se melhor execução no Grupo VV-CC. Os achados ampliam a informação sobre a contribuição da variabilidade do treinamento na emergência de comportamento criativo e adicionalmente se demonstra existência de reversibilidade e efeitos de sequenciamento.

Palavras-chave: Variabilidade no treinamento, sequência, transferência, comportamento criativo

Un hecho bien documentado en el análisis experimental de la conducta es que, a mayor variabilidad en las condiciones de entrenamiento, existen mejores posibilidades de que lo aprendido sea exitosamente transferido a situaciones novedosas (Cepeda, 1993; Cepeda, Moreno \& Larios, 2000; Irigoyen et al., 2002a; Jack, 1987; Moreno, Cepeda, Tena, Hickman \& Plancarte, 2005; Sidman, 1994; Spradlin, Cotter \& Baxley, 1973; Varela \& Quintana, 1995). Por ejemplo, estudios con procedimientos de discriminación condicional de segundo orden que han comparado los efectos de entrenar estudiantes universitarios a responder en tareas de igualación de la muestra con uno o con varios criterios de igualación (Cepeda, 1993; Irigoyen, Carpio, Jiménez, Silva, Acuña \& Arroyo, 2002b; Martínez, 2001; Varela \& Quintana, 1995) han demostrado que, tanto en el entrenamiento como en las pruebas de transferencia en las que se sustituyen las instancias de estímulo, la modalidad de estos y las relaciones-criterio, el desempeño de los individuos entrenados a responder sobre la base de múltiples criterios, es superior en comparación con los que reciben entrenamiento con un único criterio.

En términos comparativos, es mucho menor la evidencia disponible sobre la contribución que la variabilidad en las condiciones de entrenamiento que puede hacer a la emergencia de comportamiento creativo, es decir, de aquel que no consiste en 
satisfacer los criterios de ejecución que imponen las tareas experimentales, sino en generar tales criterios y estructurar nuevos problemas más que resolverlos (Carpio, 1999, 2005). Uno de los trabajos dirigidos a documentar los efectos de las condiciones variables o constantes del entrenamiento sobre el comportamiento creativo es el reportado por Carpio et al. (2006), en el que usaron procedimientos de discriminación condicional de segundo orden para comparar los efectos de dos niveles de variabilidad de las condiciones de entrenamiento, definida por el número de problemas de igualación y por el número de soluciones entrenadas, sobre el porcentaje de respuestas correctas en pruebas de transferencia y de comportamiento creativo con estudiantes universitarios. Su diseño involucró cuatro grupos de estudiantes universitarios sometidos a distintos tipos de entrenamiento: el primero de ellos (grupo C-C) fue entrenado para resolver un único problema (de identidad) con una única solución consistente en elegir siempre el mismo estímulo de comparación (ECO), que se localizaba siempre en la misma posición en la pantalla del monitor; el segundo (grupo $\mathrm{V}-\mathrm{C}$ ) fue entrenado para resolver tres problemas (de identidad, orden e inclusión), cuya solución para todos ellos siempre era igual (elegir siempre el mismo ECO que aparecía en la misma posición en la pantalla del monitor); el tercero (grupo $\mathrm{C}-\mathrm{V}$ ) fue entrenado para resolver un único problema (de identidad), cuya solución variaba ensayo a ensayo (el ECO correcto a elegir era distinto en cada ensayo y aparecía siempre en una posición distinta de la pantalla del monitor); el último (grupo V-V) fue entrenado para resolver tres problemas (de identidad, orden e inclusión), en cada uno de los cuales la solución específica variaba ensayo a ensayo (el ECO correcto era distinto en cada ensayo y aparecía en una posición distinta de la pantalla). Los resultados del estudio confirmaron que el porcentaje de respuestas correctas en el entrenamiento fue más alto en los grupos en los que solo se entrenó un único problema (C-C y $\mathrm{C}-\mathrm{V}$ ), aunque el mejor desempeño en las pruebas de transferencia fue conseguido por los grupos entrenados para resolver varios problemas ( $\mathrm{V}-\mathrm{C}$ y $\mathrm{V}-\mathrm{V})$. Asimismo, los datos revelaron que el grupo que recibió entrenamiento con varios problemas y varias soluciones (grupo $\mathrm{V}-\mathrm{V}$ ) fue el que generó correctamente un mayor porcentaje de problemas en pruebas subsecuentes de comportamiento creativo en las que los participantes estructuraban problemas no entrenados (originales) y sus criterios de solución, sin obtener por ello ningún tipo de consecuencia programada.

Aunque el estudio de Carpio, et al. (2006) documenta una relación directa entre la variabilidad del entrenamiento y la emergencia de comportamiento creativo en procedimientos de discriminación condicional, el diseño factorial empleado impuso restricciones que limitan el alcance de sus conclusiones. Específicamente, debido a que cada uno de los grupos fue expuesto exclusivamente a un solo tipo de entrenamiento, dicho estudio no pudo evaluar los posibles efectos de la exposición a más de uno de los entrenamientos y los efectos de la secuencia de exposición a estos. Esto último es relevante en al menos dos sentidos, en primer lugar; porque es prácticamente imposible que una persona reciba exclusivamente un tipo de entrenamiento en la solución de problemas durante su desarrollo conductual, y por ello es indispensable evaluar los efectos de varios tipos de entrenamiento en secuencias diferentes; en segundo lugar, debe considerarse la posible reversibilidad de los efectos identificados en el estudio referido, es decir, que después de recibir un tipo particular de entrenamiento en la solución de problemas, la exposición a un tipo distinto de entrenamiento modifique la capacidad de generar problemas que el primero pudiera haber auspiciado.

Con el objetivo de determinar la posible reversibilidad de los efectos del entrenamiento, y evaluar si la exposición a más de un tipo de entrenamiento en distintas secuencias modifica los efectos que estos tienen sobre la emergencia de comportamiento creativo en estudiantes universitarios, en 
el presente estudio se compararon los efectos de entrenar para resolver un único problema con una única solución, y después entrenar para resolver varios problemas con varias soluciones (secuencia $\mathrm{CC}-\mathrm{VV}$ ), con los efectos de entrenar en la secuencia inversa, es decir, primero entrenar para resolver varios problemas con varias soluciones y después entrenar para resolver un único problema con una única solución (secuencia VV-CC). Con este propósito, se conformaron dos grupos de participantes, uno de los cuales recibió la secuencia de entrenamiento CC-VV, mientras que el segundo fue expuesto a la secuencia VV-CC. En ambos grupos se aplicó una prueba de transferencia después de cada entrenamiento y al final una prueba de comportamiento creativo similar a la utilizada por Carpio et al. (2006).

\section{Método}

\section{Participantes}

Participaron voluntariamente cinco mujeres y cinco hombres, estudiantes del primer semestre de la carrera de Psicología de la Facultad de Estudios Superiores Iztacala (FESI) de la Universidad Nacional Autónoma de México, con edades que oscilaron entre 18 y 21 años. Todos fueron invitados a participar en el experimento informándoles solamente que se trataba de un estudio psicológico sobre aprendizaje humano y que los resultados serían anónimos. Como criterio de inclusión se estableció que no tuvieran experiencia con procedimientos de discriminación condicional.

\section{Instrumentos}

La programación y registro de eventos se realizó con un sistema de cómputo PC, empleando un programa especialmente diseñado con la aplicación SuperLab Pro, para el Sistema Operativo Windows XP.

\section{Procedimiento General}

Todas las sesiones del estudio se realizaron en las estaciones de evaluación experimental del Laboratorio de Análisis de Procesos Psicológicos Superiores de la FESI, las cuales cuentan con una silla y una mesa sobre la que se encuentra un monitor y un mouse. Los participantes solo podían manipular el mouse para desplazar el cursor en la pantalla del monitor según las instrucciones que recibían.

Una vez seleccionados, los diez estudiantes fueron asignados aleatoriamente a uno de dos grupos experimentales, según el tipo y secuencia de entrenamiento, a saber:

Grupo CC $\rightarrow$ VV: De la constancia a la variabilidad

Grupo VV $\rightarrow$ CC: De la variabilidad a la constancia

El estudio también incluyó dos pruebas subsecuentes a cada entrenamiento: una de transferencia y otra de comportamiento creativo, ambas idénticas para todos los participantes de ambos grupos. A continuación, se detallan los entrenamientos, las pruebas de transferencia y las pruebas de comportamiento creativo.

Entrenamiento CC. En esta condición se empleó un procedimiento de Discriminación Condicional de Segundo Orden, en el que cada ensayo estuvo conformado por un arreglo de siete estímulos (dibujos, letras, palabras o números) presentados en el monitor de la computadora. La distribución de los estímulos en el monitor fue la siguiente, en la parte superior se presentaron dos estímulos denominados estímulos selectores (ES's), siempre idénticos entre sí; en la parte central un estímulo denominado estímulo muestra (EM); y en la parte inferior cuatro estímulos denominados estímulos de comparación (ECO's), uno de los cuales era idéntico al EM, tal como se muestra en la figura 1. 


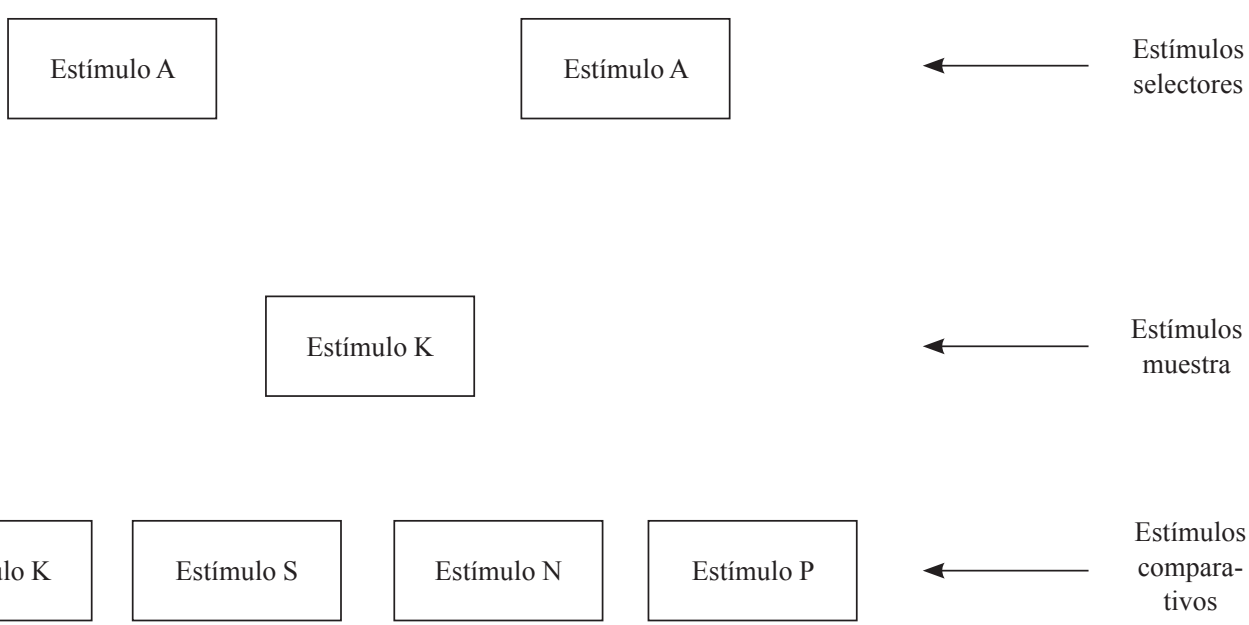

Figura 1. Distribución de los estímulos en el monitor

En total se programaron 21 ensayos de entrenamiento en esta condición. Se consideró como respuesta correcta que el sujeto oprimiera el botón izquierdo del mouse cuando el cursor estaba puesto sobre el ECO que guardaba con el EM la misma relación que entre sí guardaban los ES's (identidad). Como respuesta incorrecta se consideró oprimir el botón izquierdo cuando el cursor estaba colocado sobre cualquiera de los ECO's diferentes al ECO antes mencionado. Es importante destacar que, aunque los ES's "idénticos entre sí" cambiaban ensayo tras ensayo, el ECO correcto y el EM siempre fueron los mismos en todos los ensayos, cambiando únicamente los ECO's restantes. El ECO correcto siempre se mantuvo en la misma posición (problema constante y solución constante). Inmediatamente después de cada respuesta correcta, se retiraron de la pantalla todos los estímulos y se presentó durante tres segundos la palabra "Correcto" en el centro, al concluir los tres segundos iniciaba el siguiente ensayo. De manera similar, a cada respuesta incorrecta le seguía la palabra "Incorrecto". Las instrucciones presentadas en la pantalla al iniciar el entrenamiento fueron:
A continuación verás en la pantalla un conjunto de figuras, dos en la parte superior, una en la parte central y cuatro más colocadas en la parte inferior. Tu tarea consiste en elegir una de las figuras inferiores que se corresponda con la central, de acuerdo con la relación entre las figuras superiores. Para elegir cualquiera de las cuatro figuras inferiores, con la ayuda del mouse, pon la flecha que aparece en la pantalla sobre aquella que selecciones y da clic con el botón izquierdo. Si tu respuesta es correcta aparecerá la palabra "Correcto", de lo contrario aparecerá la palabra "Incorrecto", después aparecerá otro arreglo de figuras. Ahora presiona cualquier botón del mouse para continuar.

Entrenamiento VV. Este entrenamiento fue similar al entrenamiento $\mathrm{CC}$ antes descrito, excepto que los ensayos incluían no solamente la relación de identidad entre los ES's, sino también las de orden (O) e inclusión (IN), correspondiendo siete ensayos para cada tipo de relación, presentados en secuencia aleatoria (i.e. problema variable). Adicionalmente, todos los estímulos (ES's, EM y ECO's) cambiaban ensayo tras ensayo, de modo que la posición del 
ECO correcto varió aleatoriamente entre las cuatro posiciones posibles (i.e. solución variable). Las instrucciones que se presentaron en la pantalla al inicio del entrenamiento fueron:

A continuación verás en la pantalla un conjunto de figuras: dos en la parte superior, una en la parte central y cuatro más colocadas en la parte inferior. Tu tarea consiste en elegir una de las figuras inferiores que se corresponda con la central, de acuerdo con la relación entre las figuras superiores. Para elegir cualquiera de las cuatro figuras inferiores, con la ayuda del mouse, pon la flecha que aparece en la pantalla sobre aquella que selecciones y da clic sobre ella con el botón izquierdo. Si tu respuesta es correcta aparecerá la palabra "Correcto", de lo contrario aparecerá la palabra "Incorrecto", después aparecerá otro arreglo de figuras. Ahora, presiona cualquier botón del mouse para continuar.

Prueba de Transferencia. Inmediatamente después de cada entrenamiento, todos los participantes fueron expuestos a una prueba de transferencia de 21 ensayos, cuyo procedimiento general fue semejante al utilizado en el entrenamiento VV, con las siguientes diferencias:

- Ninguna respuesta (correcta o incorrecta) tuvo consecuencias programadas, excepto dar lugar al siguiente ensayo.

- Se sustituyeron todos los estímulos usados en el entrenamiento por estímulos novedosos.

- Se agregaron las relaciones de semejanza (S), diferencia (D), menor que (MEQ) y exclusión (EX) entre los ES's, de manera que ahora el ECO correcto podía ser el que guardara con el EM cualquiera de estas relaciones, dependiendo de cuál de ellas existía entre los ES's del ensayo correspondiente.

- A cada uno de los tipos de relación (ID, O, IN, $\mathrm{S}, \mathrm{D}, \mathrm{MEQ}, \mathrm{EX}$ ) correspondieron tres ensayos, distribuidos aleatoriamente en la prueba.
- Los estímulos empleados en las dos pruebas de transferencia (una después de cada entrenamiento) fueron diferentes entre sí.

Las instrucciones que se presentaron en la pantalla al inicio de esta prueba fueron:

A continuación verás en la pantalla un conjunto de figuras, dos en la parte superior, una en la parte central y cuatro más colocadas en la parte inferior. Tu tarea consiste en elegir una de las figuras inferiores que se corresponda con la central, de acuerdo con la relación entre las figuras superiores. Para elegir cualquiera de las cuatro figuras inferiores, pon la flecha del mouse sobre aquella que selecciones y da clic con el botón izquierdo. En esta ocasión no se te informará si tu respuesta ha sido correcta o incorrecta. Ahora presiona cualquier botón del mouse para continuar.

Prueba de Comportamiento Creativo. Al finalizar la prueba de transferencia, todos los participantes recibieron un cuadernillo con siete hojas, en las cuales se presentaba un arreglo similar a los empleados en las condiciones anteriores, pero que difería en que el espacio correspondiente a los ES's estaba vacío y con uno de los ECO's señalado con un asterisco. En cada hoja, se solicitaba al participante que completara los arreglos y explicara por qué lo había hecho de ese modo. En estas pruebas no existía una relación-criterio prestablecida entre los estímulos selectores que determinara la relación EM-ECO definitoria del ECO correcto en cada ensayo. Por el contrario, al ser el propio participante el que elaboraba los ES's y su relación, no estaba resolviendo un problema, sino creándolo. Un análisis posterior del tipo de problemas (i.e. relaciones entre ES's) construidos por cada participante permitió determinar si estos fueron similares a los que resolvieron en el entrenamiento, o bien eran novedosos, considerando que en este último caso se estaba frente a un caso de comportamiento 
creativo. En la figura 2 se muestra un ejemplo de las hojas de la prueba de comportamiento creativo.

Al entregar el cuadernillo, se pidió a los participantes que dibujaran en los espacios vacíos correspondientes a los ES's, las figuras, palabras o números que hicieran que el ECO señalado con el asterisco fuera correcto. Adicionalmente, se les pidió que escribieran la razón de ello. Cada uno de los siete arreglos presentados correspondió a una de las relaciones empleadas durante la prueba de transferencia (ID, O, IN, S, D, MEQ, EX). No se impuso ningún límite de tiempo para la realización de la prueba. Con propósitos de identificación y análisis, la elaboración de los dibujos fue denominada "respuesta instrumental" y la explicación "respuesta referencial".

\section{Secuencia de entrenamientos para cada grupo.} La secuencia de condiciones para el grupo CC-VV fue entrenamiento $\mathrm{CC}$, primera prueba de transferencia y primera prueba de comportamiento creativo, seguida de entrenamiento VV, segunda prueba de transferencia y segunda prueba de comportamiento creativo. De manera complementaria, la secuencia de condiciones para el grupo VV-CC fue entrenamiento $\mathrm{VV}$, primera prueba de transferencia y primera prueba de comportamiento creativo seguida de entrenamiento $\mathrm{CC}$, segunda prueba de transferencia y segunda prueba de comportamiento creativo.

\section{Resultados}

A continuación, se presentan los resultados, comparando entre grupos los porcentajes de respuestas correctas en los entrenamientos y las pruebas de transferencia, así como los porcentajes de problemas generados y correctamente referidos en las pruebas de comportamiento creativo.

\section{Entrenamientos}

En la figura 3 se presentan los porcentajes promedio de respuestas correctas obtenidos por cada

Por favor completa los arreglos colocando en los recuadros superiores las figuras, palabras o números, de modo que la figura, palabra o número señalada con un asterisco corresponda con la del centro y explica por qué:

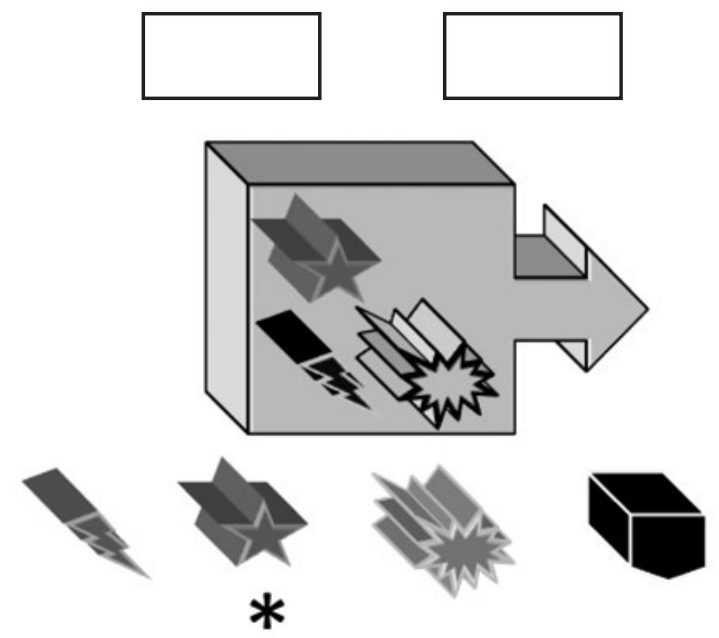

¿POR QUÉ?:

Figura 2. Ejemplo de las hojas de la prueba de comportamiento creativo 


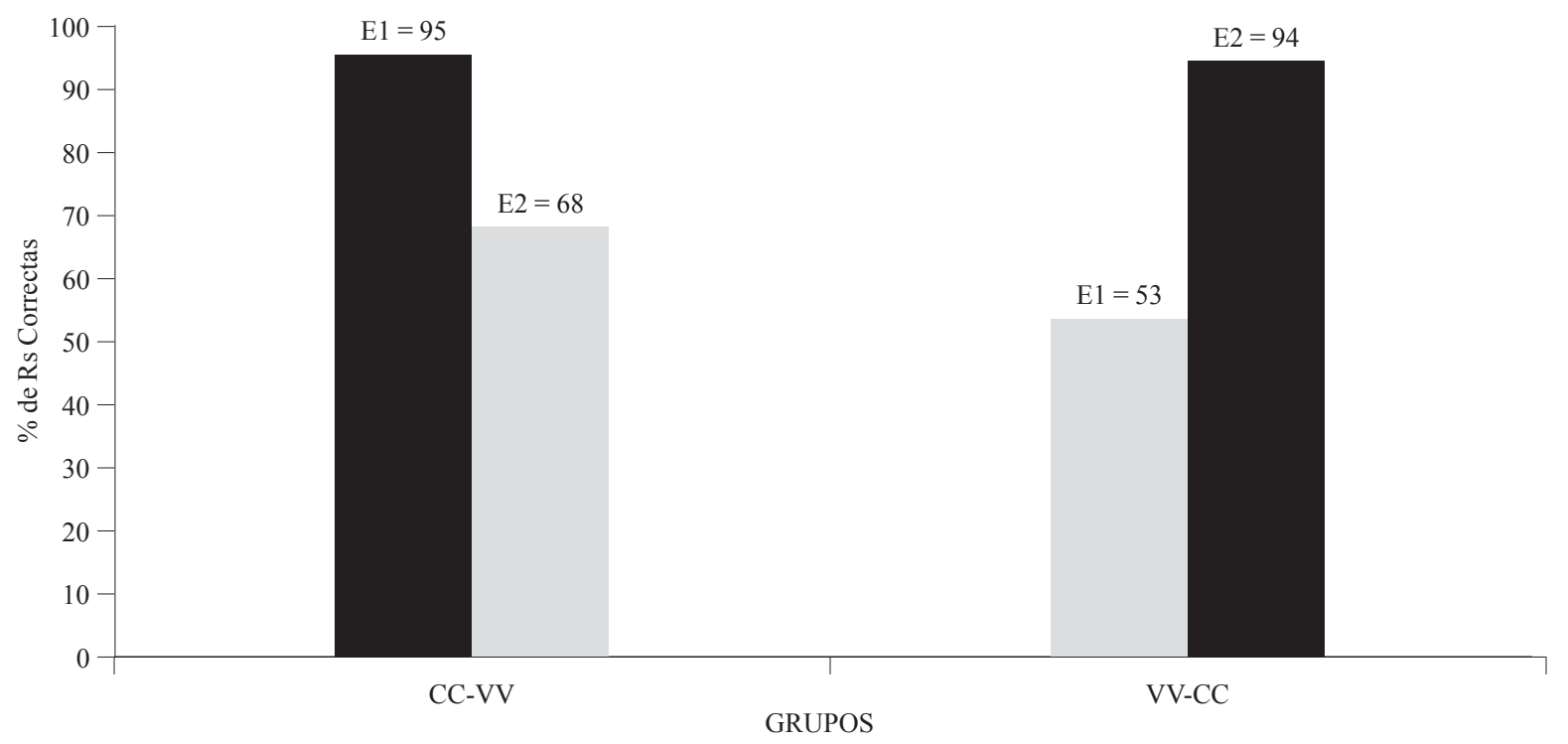

Figura 3. Porcentaje de respuestas correctas en entrenamientos 1 (E1) y 2 (E2)

uno de los grupos en los distintos entrenamientos. En esta se aprecia con claridad que en el entrenamiento a resolver un único problema con una misma solución (entrenamiento CC), ambos grupos alcanzaron porcentajes de respuestas correctas muy elevados, independientemente de que fuera el primero o el segundo entrenamiento recibido. Asimismo, se aprecia que en el entrenamiento a resolver varios problemas con diversas soluciones (entrenamiento VV), ambos grupos obtuvieron porcentajes de respuestas correctas mucho más bajos, independientemente del orden en que fueron realizados, es decir, al margen de que el entrenamiento VV hubiera sido el primero o el segundo que recibieron los participantes. Adicionalmente, la figura 3 permite apreciar que no existen diferencias de importancia entre los porcentajes de respuestas correctas de ambos grupos en el entrenamiento CC, así como tampoco existen diferencias de importancia de los porcentajes de respuestas correctas durante el entrenamiento VV. El análisis de varianza de factores fijos - factor $1=$ valor ordinal del entrenamiento (primero contra segundo); factor 2= tipo de entrenamiento (CC contra VV) aplicado a los datos con el paquete SPSS (versión 11.5)— arrojó un valor de F significativo para la interacción de los factores $F(1,16)=27213, p<0.05$ y no significativo para los efectos principales de cada uno de los factores por separado $F(1,16)=1344, p>0.05$ para factor 1 , y $F(1,16)=1029, p>0.05$ para factor 2 , lo cual confirmó que la varianza observada en los grupos fue superior a la esperada por azar y dependiente de la interacción del tipo de entrenamiento con la secuencia recibida.

Dado que solo hubo dos grupos pequeños $(n=5)$ por cada uno de los factores, no fue posible aplicar pruebas post-hoc, y al no tener condiciones para garantizar la igualdad de varianza, los supuestos de normalidad y la homocedasticidad en las muestras, la revisión posterior de los datos se limitó a la inspección visual, revelándose una diferencia importante entre ambos tipos de entrenamiento dentro de cada grupo, aunque al comparar los entrenamientos CC y VV entre grupos no se aprecian grandes diferencias.

\section{Pruebas de transferencia}

La figura 4 presenta el porcentaje promedio de respuestas correctas en cada una de las pruebas de 


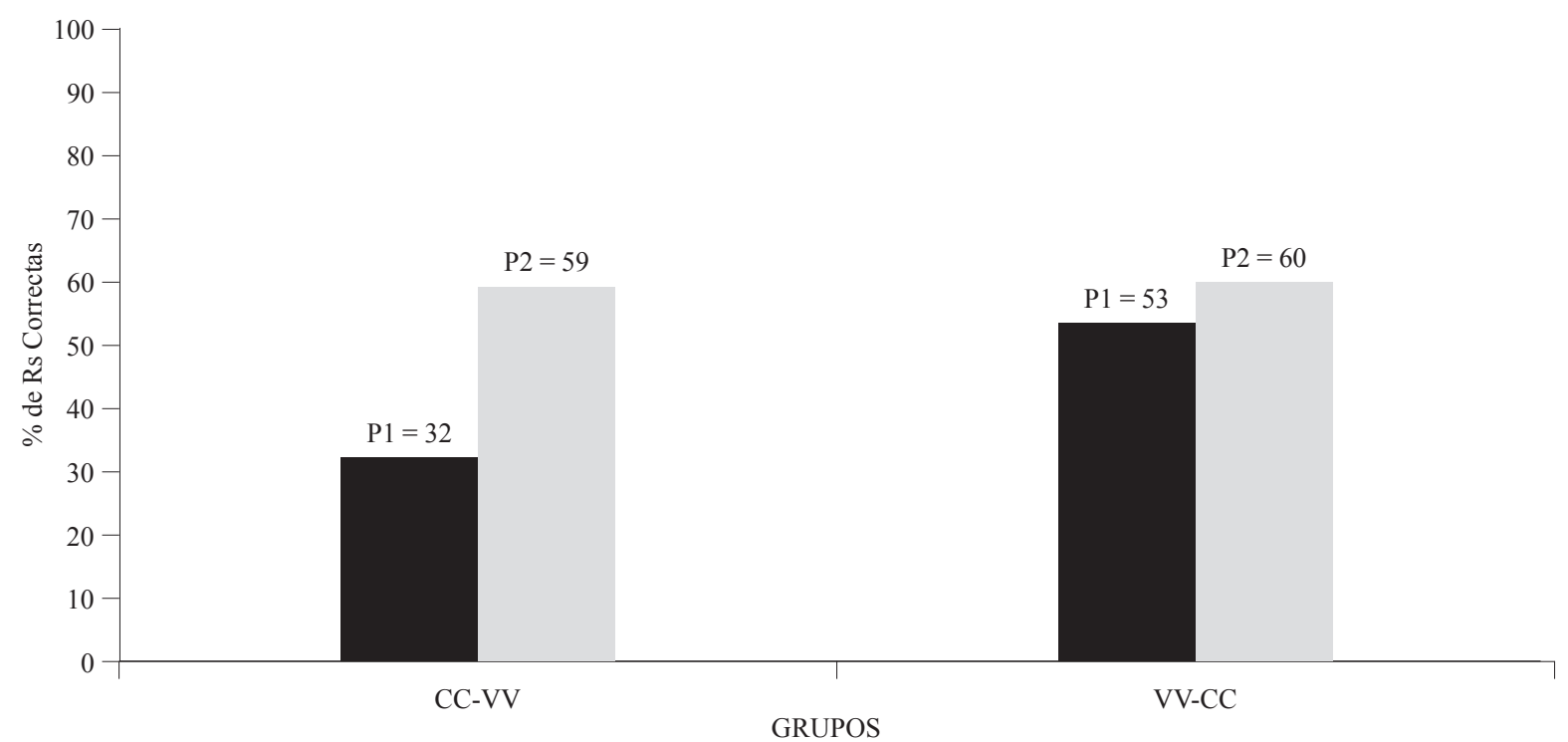

Figura 4. Porcentaje de respuestas correctas en pruebas 1 (P1) y 2 de transferencia (P2)

transferencia para cada grupo, en ella se aprecia que en la primera prueba de transferencia el porcentaje de respuestas correctas del grupo CC-VV es notablemente más baja que en el grupo VV-CC, aunque en la segunda prueba, dicho porcentaje es prácticamente el mismo en ambos grupos. El análisis de varianza aplicado a estos datos con el paquete SPSS (versión 11.5) arrojó un valor de $F(1,16)$ $=35244, p<0.5$, y la prueba post-hoc de Tuckey aplicada subsecuentemente reportó diferencias significativas entre los porcentajes de respuestas correctas en la primera prueba del grupo CC-VV, y la segunda prueba aplicada a este mismo grupo, así como con los porcentajes obtenidos en ambas pruebas por el grupo VV-CC.

La figura 5 reúne los porcentajes de respuestas correctas en cada una de las condiciones del estudio para ambos grupos, en ella puede apreciarse fácilmente que la ejecución en los entrenamientos recibidos por ambos grupos dependió de su tipo y no de su posición en la secuencia (primero o segundo), mientras que la ejecución en las pruebas de transferencia sí fue afectada tanto por el entre- namiento inmediato anterior, como por su posición en la secuencia.

Al desglosar los resultados de las pruebas de transferencia en términos de porcentajes de problemas semejantes a los entrenados, y porcentajes de problemas completamente novedosos (no entrenados) que se resolvieron correctamente en cada una de dichas pruebas, fue posible apreciar que en ambas pruebas los dos grupos obtuvieron porcentajes más altos de respuestas correctas ante los problemas ya entrenados que ante los no entrenados (figura 6).

\section{Prueba de comportamiento creativo}

La intención fundamental del presente estudio fue determinar si diferentes secuencias de exposición a los entrenamientos VV y CC afectaban de modo distinto la emergencia de comportamiento creativo en estudiantes universitarios, al utilizar procedimientos de discriminación condicional de segundo orden. De ser afirmativa la respuesta a la pregunta experimental propuesta, en la prueba 


\section{Claudio Carpio, Uirginia Pacheco, Germán morales, Rosalinda Arroyo y Linda Pacheco-Lechón}

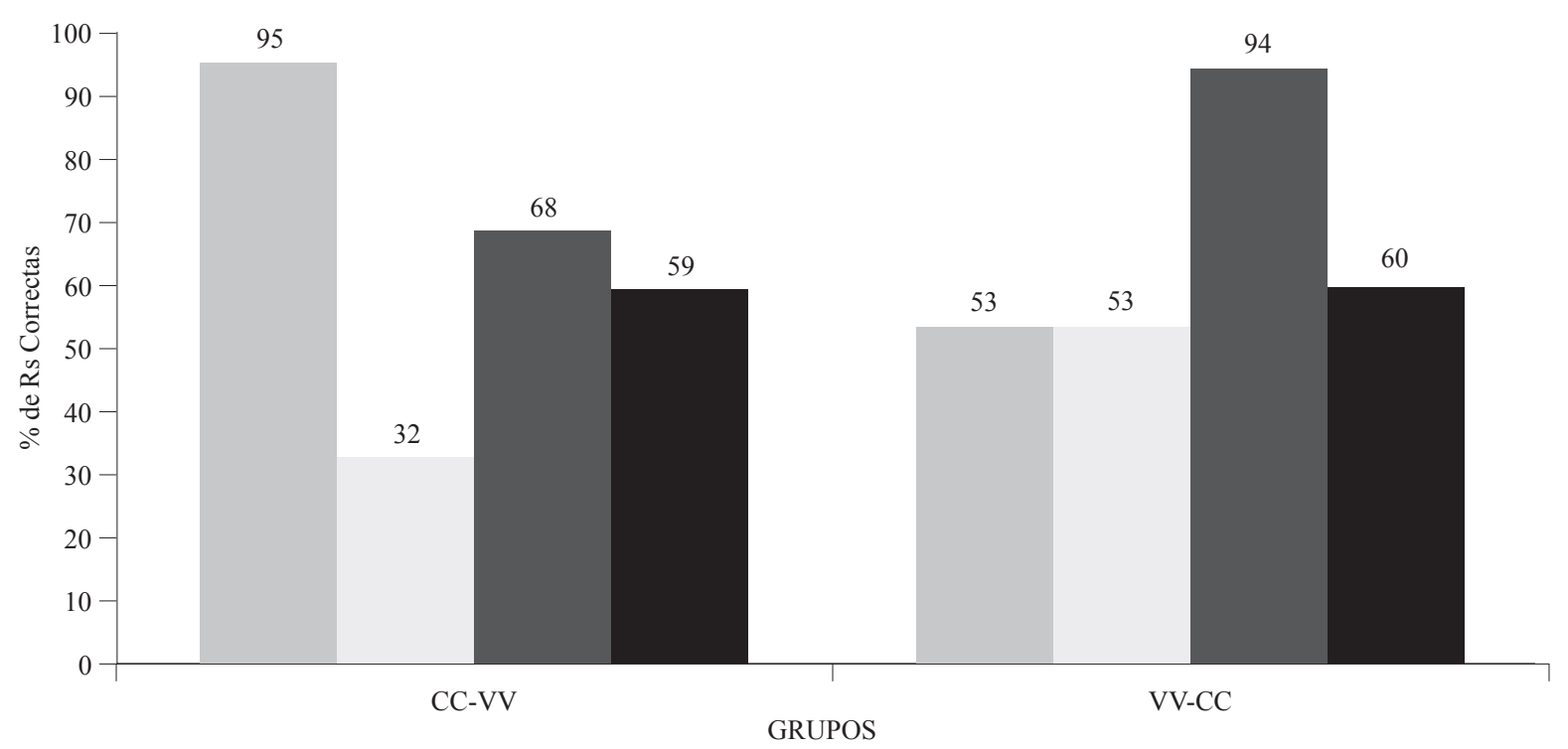

Figura 5. Porcentaje de respuestas correctas por condición

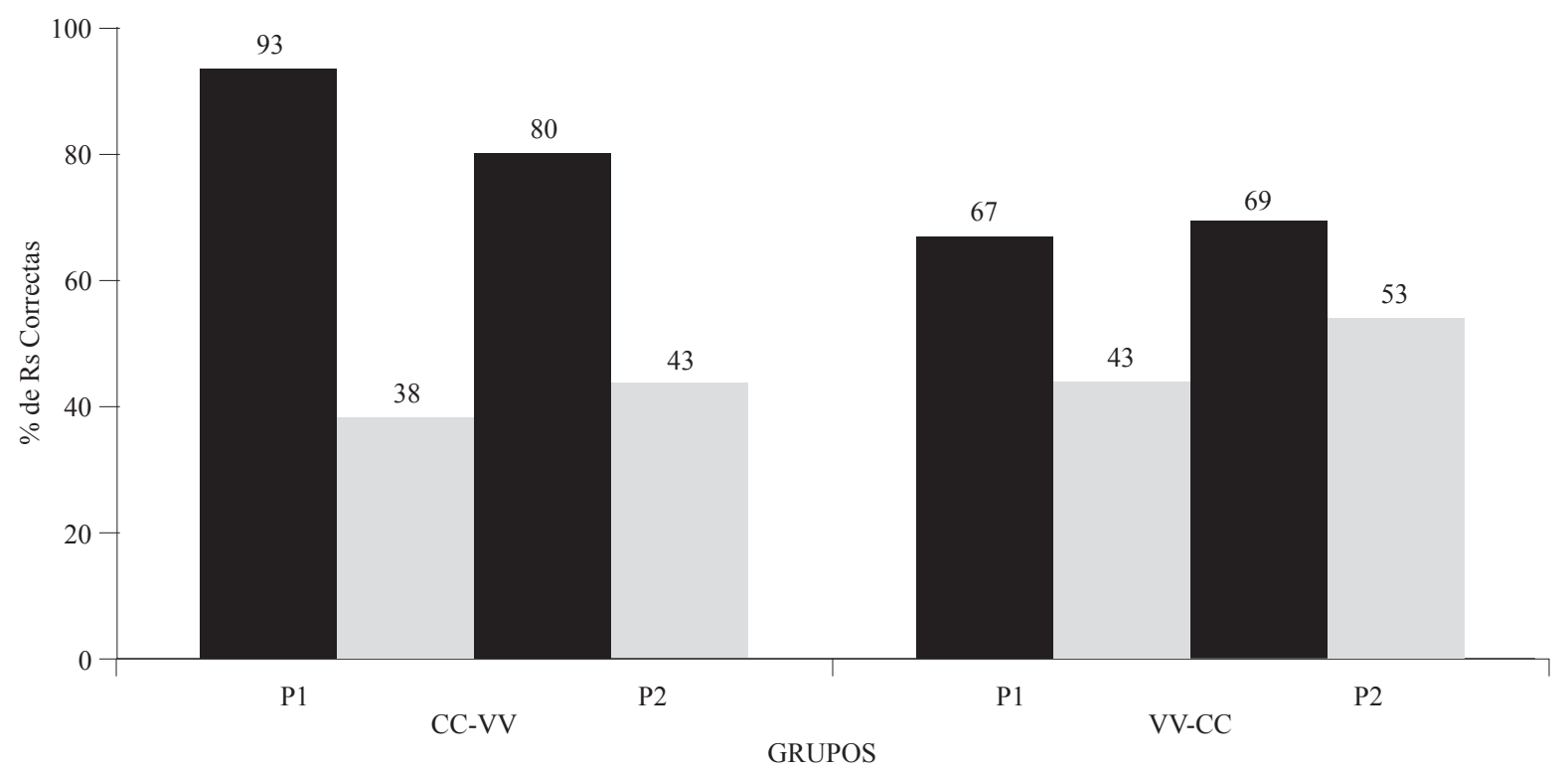

Figura 6. Porcentaje de respuestas correctas entrenadas (barra oscura) y no entrenadas (barra blanca) en la prueba 1 (P1) y la prueba 2 (P2) de transferencia

de comportamiento creativo deberían existir diferencias significativas entre los porcentajes de problemas correctamente elaborados por cada uno de los grupos en la prueba de comportamiento creativo, en caso contrario, es decir, que no existieran tales diferencias, la conclusión del estudio habría de orientarse en el sentido de que la secuencia de exposición no afecta la emergencia de comportamiento creativo.

La figura 7 presenta los porcentajes promedio de problemas correctamente elaborados por cada uno de los grupos, observándose que no existe 


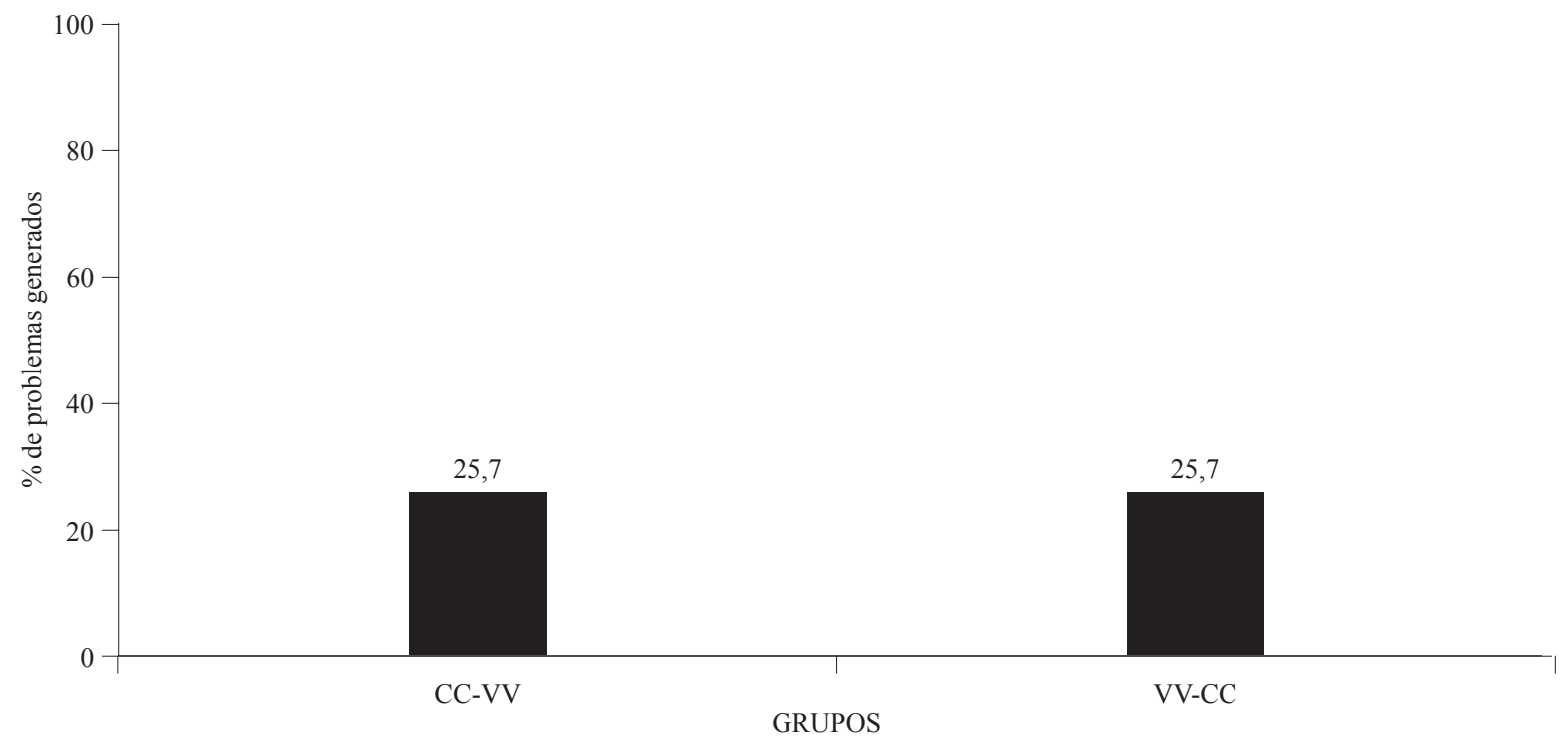

Figura 7. Porcentaje de problemas generados en la prueba de comportamiento creativo

absolutamente ninguna diferencia entre ambos grupos, pues los dos alcanzaron exactamente el mismo porcentaje.

Al analizar en qué medida los problemas generados en la prueba de comportamiento creativo eran similares a los entrenados, o qué tanto eran problemas novedosos, se encontró que en ambos grupos los problemas generados fueron mayoritariamente semejantes a los entrenados, aunque en el grupo CC-VV los problemas novedosos fueron aproximadamente el $33 \%$ del total, mientras que en el grupo VV-CC, estos alcanzaron casi el $45 \%$ (figura 8).

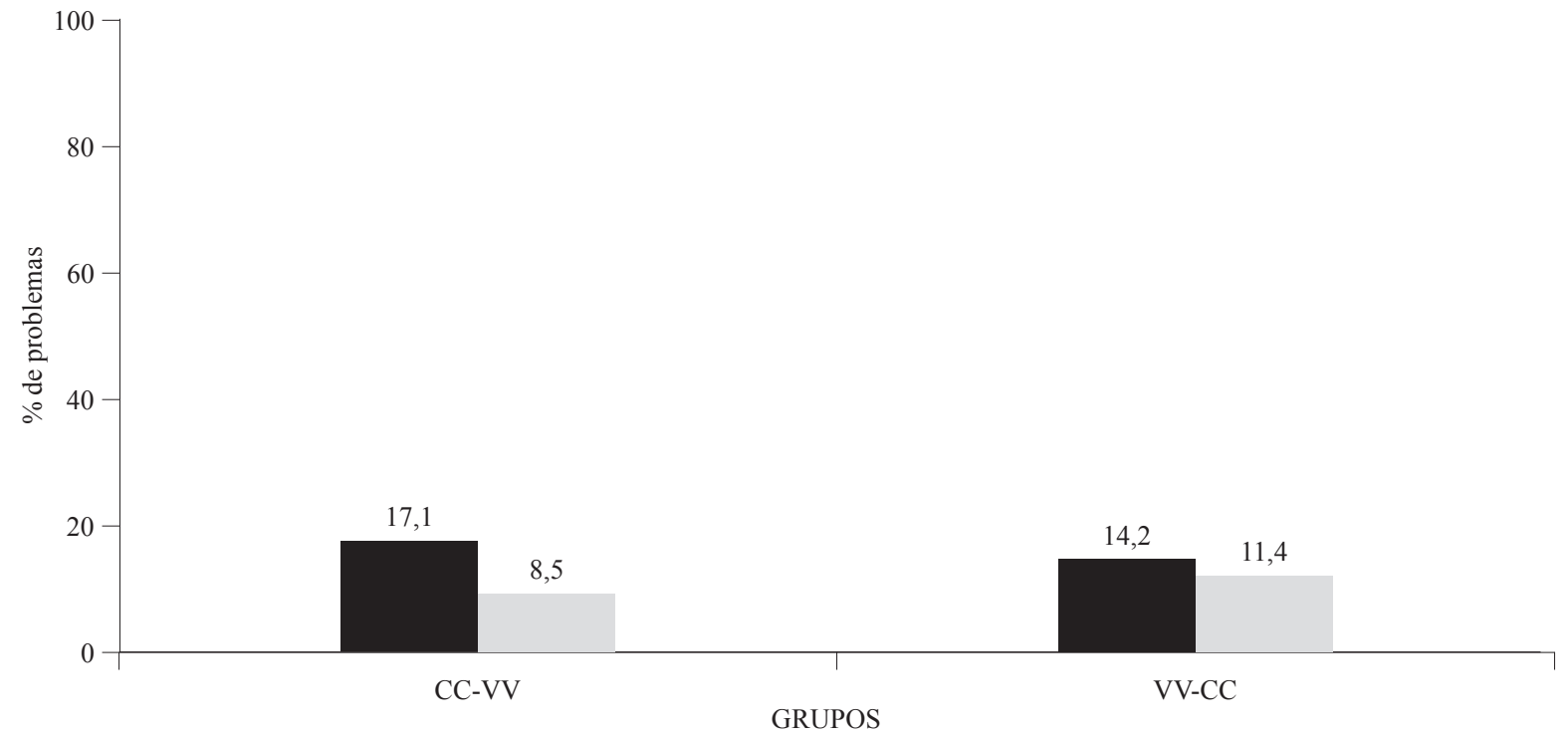

Figura 8. Porcentaje de problemas entrenados (barra oscura) y no entrenados (barra blanca) generados en la prueba de comportamiento creativo 
Un último aspecto considerado en el estudio fue el relativo al porcentaje de referencias correctas realizadas a los problemas generados en la prueba de comportamiento creativo. La figura 9 presenta este dato y en ella se observa una ligera superioridad del grupo CC-VV, respecto al grupo VV-CC, con una diferencia menor al $10 \%$.

\section{Discusión}

Los datos obtenidos en el presente estudio son relevantes no solo para el problema central de la investigación, sino también para otros que amplían las perspectivas para futuros trabajos. En primer lugar, es importante subrayar que haber encontrado porcentajes de respuestas correctas significativamente más elevados en el entrenamiento CC que en el entrenamiento VV es plenamente consistente con los hallazgos de Carpio et al. (2006) y de Cepeda (1993), y confirma que la invarianza de la relación entre el problema y la actividad que lo resuelve facilita el aprendizaje en condiciones de constancia máxima en el entrenamiento.

Este dato también documenta, adicionalmente, que el desempeño en ambos tipos de entrenamiento (CC y VV) es independiente de la secuencia en que estos son recibidos, siendo, en consecuencia, las características y propiedades funcionales de cada uno de ellos las que determinan la ejecución, lo que fortalece la idea de reversibilidad de los efectos conductuales de cada procedimiento de entrenamiento. No sobra señalar que este resultado previene sobre cualquier creencia de irreversibilidad de los eventuales efectos nocivos de procesos educativos inadecuados (Skinner, 1968).

Un segundo aspecto relevante de los resultados consiste en la superioridad de la transferencia de lo aprendido bajo condiciones variables de entrenamiento, respecto a la observada después del entrenamiento bajo condiciones constantes de entrenamiento. Este hecho es consistente con lo reportado por otros autores (Cepeda, 1993; Cepeda, Moreno \& Larios, 2000; Irigoyen et al., 2002a; Jack, 1987; Moreno, Cepeda, Tena, Hickman \& Plancarte, 2005; Sidman, 1994; Spradlin, Cotter \& Baxley, 1973; Varela \& Quintana, 1995), aunque también permite especificar que esto es así solo cuando el entrenamiento $\mathrm{CC}$ es el primer entrenamiento recibido. Como se observó, si primero se recibe un entrenamiento $\mathrm{VV}$ y posteriormente un entrenamiento $\mathrm{CC}$, entonces la transferencia que sigue a este es tan buena como la que se observa

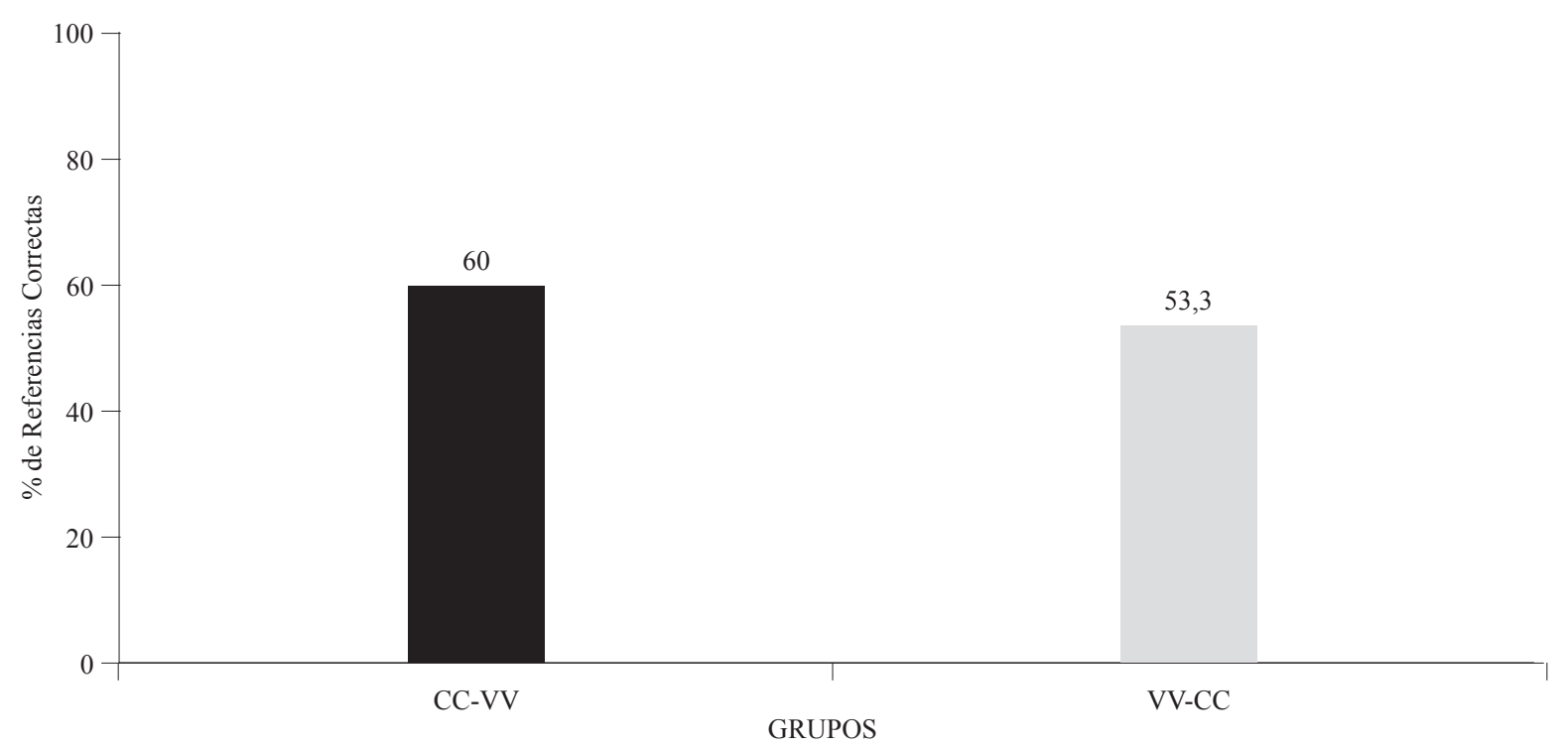

Figura 9. Porcentaje de referencias correctas en la prueba de comportamiento creativo 
después del entrenamiento tipo VV. Esta precisión es importante, pues ayuda a entender que los efectos del entrenamiento sobre la transferencia no son absolutos sino relativos a su orden secuencial, lo cual también obliga a pensar en las secuencias apropiadas durante la planeación de la enseñanza y el aprendizaje en instituciones educativas (Ribes, 2002).

Un tercer hecho que sobresale, a partir de los resultados obtenidos, es que en las pruebas de transferencia la ejecución se mantuvo con buenos niveles de efectividad ante nuevas instancias de estímulo, siempre y cuando entre ellos existan las mismas relaciones que estuvieron vigentes en el entrenamiento, lo cual deja ver que no basta el entrenamiento en condiciones variadas (entrenamiento VV) para generar transferencia hacia relaciones no entrenadas, y plantea la necesidad de explorar otros factores que pudieran propiciarla (entre ellas el entrenamiento por instrucción y no solo por consecuencias). No puede obviarse en este contex to que entre las condiciones de entrenamiento sobresalen las instrucciones al inicio de cada procedimiento, las cuales modulan diferencialmente el contacto del participante con aspectos específicos de la tarea (estímulos o relaciones), y son estos los que ganan control sobre la ejecución tanto en esa como en subsecuentes tareas o pruebas. Esta interpretación, sin embargo, debe ser evaluada experimentalmente en estudios que analicen los efectos de distintos tipos de instrucciones sobre la adquisición y transferencia a problemas novedosos, pero también sobre la emergencia de comportamiento creativo.

Además de lo anterior, es necesario destacar que si bien es cierto que en la segunda prueba de transferencia ya no había diferencias entre los grupos, y que el porcentaje de problemas generados en la prueba de comportamiento creativo fue el mismo en ambos grupos, la composición de este porcentaje es diferente en cada uno de ellos. En el grupo $\mathrm{CC}-\mathrm{VV}$ los problemas novedosos generados fueron menos que en el grupo VV-CC, lo cual sugiere un comportamiento creativo superior en este último grupo. A su vez, esto hace evidente la necesidad de considerar que la emergencia del comportamiento creativo no es repetición de lo aprendido, sino la creación de problemas novedosos, originales, no entrenados. El hecho de que en el grupo CCVV fuera mayor el porcentaje de problemas ya entrenados, los que se generaron en la prueba de comportamiento creativo, sugiere, otra vez, que la secuencia de exposición a las condiciones de entrenamiento aquí evaluadas sí tiene un efecto que no debe soslayarse en el estudio de las condiciones promotoras de esa forma de comportamiento. Esto también debe considerarse en la planeación educativa de condiciones de enseñanza-aprendizaje.

Por último, si bien debe reconocerse la necesidad de avanzar en el desarrollo de estrategias más precisas para evaluar el efecto de las condiciones variables o constantes de entrenamiento, ampliando los controles metodológicos (incluyendo pruebas de comportamiento creativo previas al entrenamiento), también puede aceptarse que el conjunto de resultados del presente estudio contribuye a confirmar los efectos que la variabilidad del entrenamiento tiene sobre la adquisición de desempeños efectivos en la solución de problemas de igualación y su transferencia a situaciones novedosas. Específicamente, confirman que se aprende más rápidamente la solución de los problemas cuando estos se mantienen constantes, aunque el ajuste exitoso a problemas distintos es mejor favorecido por el entrenamiento en situaciones de mayor variabilidad en el tipo de problemas y sus soluciones (Cepeda et al., 2000; Cepeda, 1993; Irigoyen et al., 2002; Moreno et al., 2005; Varela \& Quintana, 1995). El estudio también amplía la información sobre la contribución que hace la variabilidad del entrenamiento a la emergencia de comportamiento creativo y la reversibilidad de sus efectos. En particular, demuestran que sí existe un efecto de la secuencia de exposición a las condiciones constantes y variables del entrenamiento, resultando más favorable a la emergencia de comportamiento creativo la secuencia VV-CC. Futuras investigaciones pueden 
concentrarse, como se ha sugerido, en la contribución de las instrucciones y su interacción con las consecuencias programadas en el entrenamiento.

\section{Referencias}

Carpio, C. (1999). La creatividad como conducta. En A. Bazán (Ed.), Aportes Conceptuales y Metodológicos en Psicología Aplicada (pp. 37-54). México: ITSON.

Carpio, C. (2005). Condiciones de entrenamiento que promueven comportamiento creativo: Un análisis experimental con estudiantes universitarios (Tesis doctoral, Universidad Iberoamericana, México).

Carpio, C., Silva, H., Landa, E., Morales, G., Arroyo, R., Canales, C., \& Pacheco, V. (2006). Generación de criterios de igualación: Un caso de conducta creativa. Universitas Psychologica, 5(1), 127-138.

Cepeda, M. (1993). Efectos de la variabilidad en criterios de entrenamiento sobre pruebas de transferencia y formulación de reglas (Tesis de maestría, Universidad Nacional Autónoma de México, México).

Cepeda, M., Moreno, D., \& Larios, R.(2000). Relación de un entrenamiento variado con opciones textuales y la transferencia en una tarea de discriminación condicional. Revista Psicología y Ciencia Social, 2(4), 3-16.

Irigoyen, J., Carpio, C., Jiménez, M., Silva, H., Acuña, K., \& Arroyo, A. (2002a). Efectos de los diferentes tipos funcionales de retroalimentación y su presentación parcial en el entrenamiento y transferencia de desempeños efectivos. Revista sonorense de psicología, 16(1 y 2), 35-43.

Irigoyen, J., Carpio, C., Jiménez, M., Silva, H., Acuña, K., \& Arroyo, A. (2002b). Variabilidad en el entrenamiento con retroalimentación parcial en la adquisición de desempeños efectivos y su transferencia. Enseñanza e investigación en Psicología, 7(2), 221-234.

Jack, A. (1987). Historical review and appraisal of research on the learning, retention and transfer of human motor skills. Psychological Bulletin, 101(1), 41-74.

Martínez, H. (2001). Estudios sobre transferencia en comportamiento humano. En G. Mares \& Y. Guevara (Eds.), Psicología Interconductual. Avances en la investigación básica (pp. 37-58). México: Universidad Nacional Autónoma de México.

Moreno, D., Cepeda, L., Tena, O., Hickman, H., \& Plancarte, P. (2005). Conducta gobernada por reglas: implicaciones educativas. En C. Carpio \& J. J. Irigoyen (Eds.), Psicología y educación. Aportaciones desde la Teoría de la Conducta (pp. 175-212). México: Universidad Nacional Autónoma de México.

Ribes, E. (2002). Psicología del aprendizaje. México: Manual moderno.

Varela, J., \& Quintana, C. (1995). Comportamiento inteligente y su transferencia. Revista Mexicana de Análisis de la Conducta, 21(1), 47-66.

Sidman, M. (1994). Equivalence relations and behavior: A research story. Boston, MA: Authors Cooperative.

Skinner, B. F. (1968). The technology of teaching. New York: Appleton-Century-Crofts.

Spradlin, J. E., Cotter, V. W. \& Baxley, N. (1973). Establishing a conditional discrimination without direct training: a study of transfer with retarded adolescents. American Journal of Mental Deficiency, 77(5), G 556-566. 